\title{
RHIC Injection Kicker Impedance
}

\author{
V. Mane, S. Peggs, D. Trbojevic, W. Zhang \\ Brookhaven National Laboratory, * \\ Upton, NY 11973, USA
}

\section{Introduction}

The longitudinal impedance of the RHIC injection kicker is measured using the wire method upto a frequency of $3 \mathrm{GHz}$. The mismatch between the $50 \mathrm{ohm}$ cable and the wire and pipe system is calibrated using the TRL calibration algorithm. Various methods of reducing the impedance, such as coated ceramic pipe and copper strips are investigated.

\section{Kicker Parameters}

The RHIC injection kicker is a pulsed transmission line kicker, consisting of 15 ferrite sections and 14 ceramic sections. The length of the kicker is $1.1 \mathrm{~m}$ and there are 4 such units in each ring. Figure 1 shows the kicker cross section with the outer conductor, C-shaped ferrite, inner conductor and the ceramic beam pipe [1]. The design characteristic impedance of the transmission line kicker is $25 \mathrm{ohm}$. Each kicker will be pulsed with a Blumlein pulser and terminated by a matched resistor. The ferrite material used is Ceramic Magnetics CMD5005. The initial permeability of the ferrite is 1600 at low frequencies, and the dielectric constant is 12 . The ceramic is manufactured by Trans-Tech and has a high dielectric constant of 100 . The wave propagation velocity in the magnet is approximately $1 / 15$ the speed of light. The design field risetime in the magnet is $80 \mathrm{nsec}$, and the flattop is about $40 \mathrm{nsec}$. The nominal integrated magnetic field is 1900 G-m from 4 units [2].

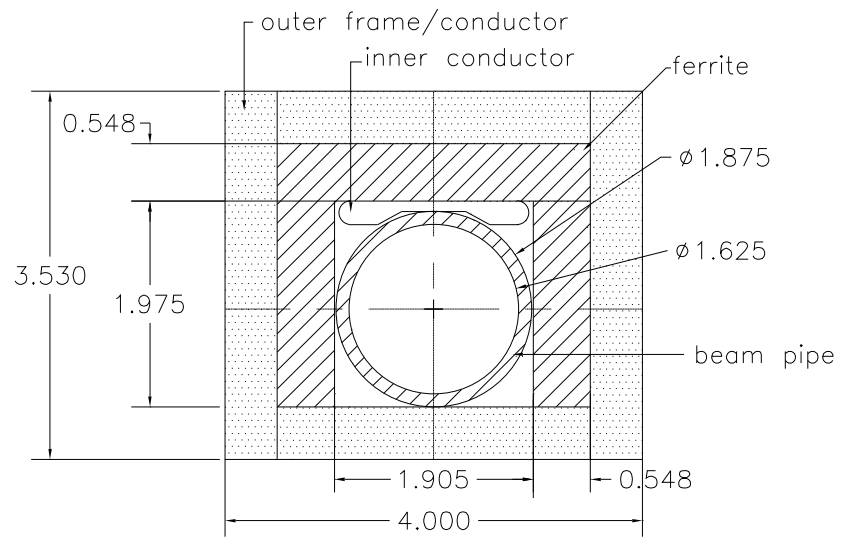

Figure. 1. RHIC injection kicker cross section, Dimensions in inches

\section{Test Setup}

Figure 2 gives a plot of the experimental setup. The kicker is placed between two rectangular side pipes with the same dimension as the outer conductor of the kicker. The wire is placed on

\footnotetext{
* Operated by Associated Universities Incorporated, under contract with the U.S. Department of Energy.
}

the axis of the ceramic beam pipe. The characteristic impedance of the wire and rectangular pipe system is $280 \mathrm{ohm}$. The system is connected to the Network Analyzer through a $50 \mathrm{ohm}$ cable. In order to calibrate the mismatch between the $50 \mathrm{ohm}$ cable and the wire-pipe system, the following set of measurements are performed

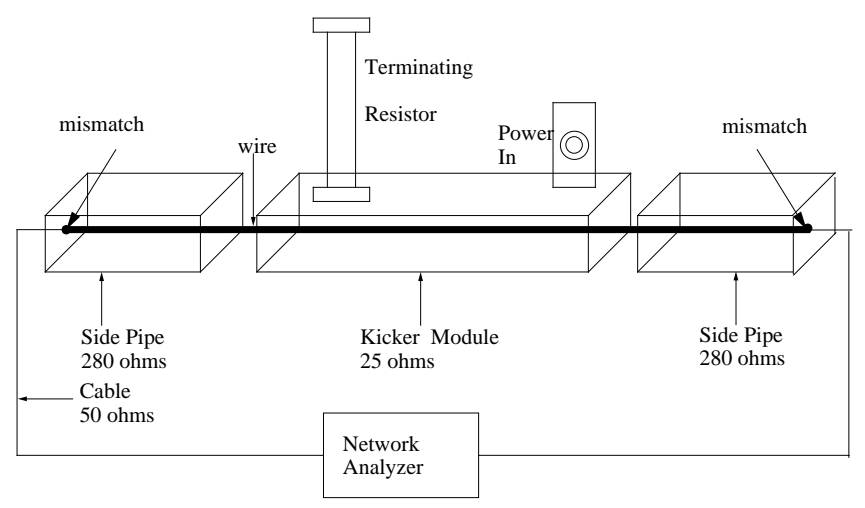

Figure. 2. Bench Setup for Wire Measurement

Through Measurement: The side pipes are connected directly to each other.

Reflect Measurement: A reflective load is connected to each side pipe.

Line (Delay) Measurement: A straight pipe of arbitrary length is connected between the side pipes. Measurement is done with two pipes of different lengths.

Reference Measurement: The device is replaced by a reference pipe of the same length.

The kicker is then calibrated using the TRL calibration algorithm [3]. The impedance is obtained from the transmission coefficient $S_{21}$ using the following relation

$$
Z(\omega)=2 Z_{c} \frac{\left(S_{21}(r e f)-S_{21}(D U T)\right)}{S_{21}(D U T)}
$$

where $Z_{c}$ is the characteristic impedance of the reference pipe, $S_{21}(D U T)$ is the transmission coefficient of the Device Under Test (DUT) and $S_{21}(r e f)$ is the transmission coefficient of the reference pipe.

\section{Measurement Results}

The kicker impedance has to be measured carefully, as several factors contribute to the complexity of the impedance. If the 
kicker outer conductor is isolated from the beam pipe, return currents flow outside the outer conductor, causing the beam to see the external environment. Therefore the outer conductor of the kicker is grounded to the beampipe. To simulate this in the measurement setup, the side pipes are electrically connected to the outer conductor of the kicker. The cables from the Blumlein power supply to the kicker are $75 \mathrm{~m}$ long and are open when the kicker is switched off. The bench measurements are performed, with cables of length $15.5 \mathrm{~cm}$. Some measurements were also done with $40.5 \mathrm{~cm}$ long cables and $75 \mathrm{~m}$ long cable. The length of the cable does not seem to have any effect on the impedance of the magnet. The measurements presented in this paper were done on a half length prototype $(60 \mathrm{~cm}$ long $)$.

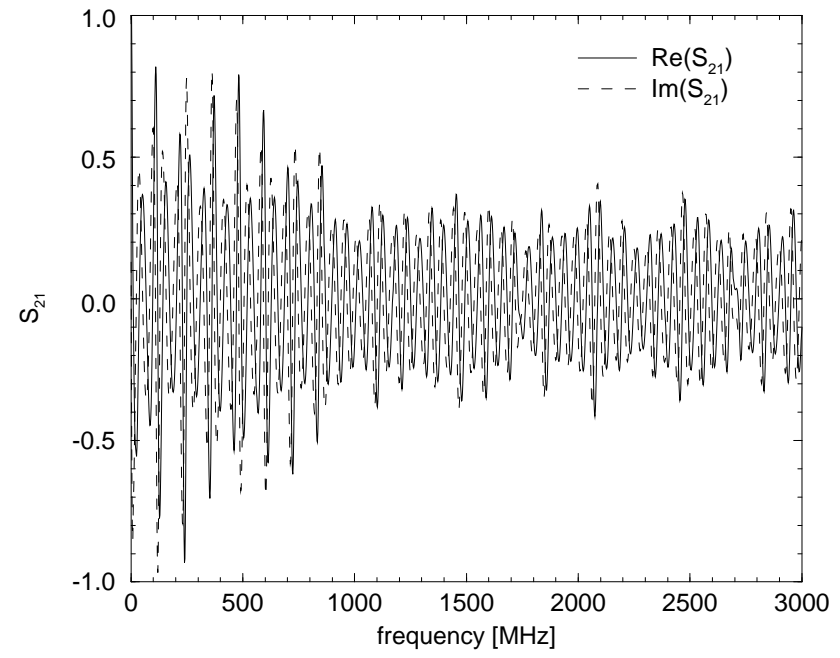

Figure. 3. Uncalibrated $S_{21}$ Parameter of the Kicker Half Module

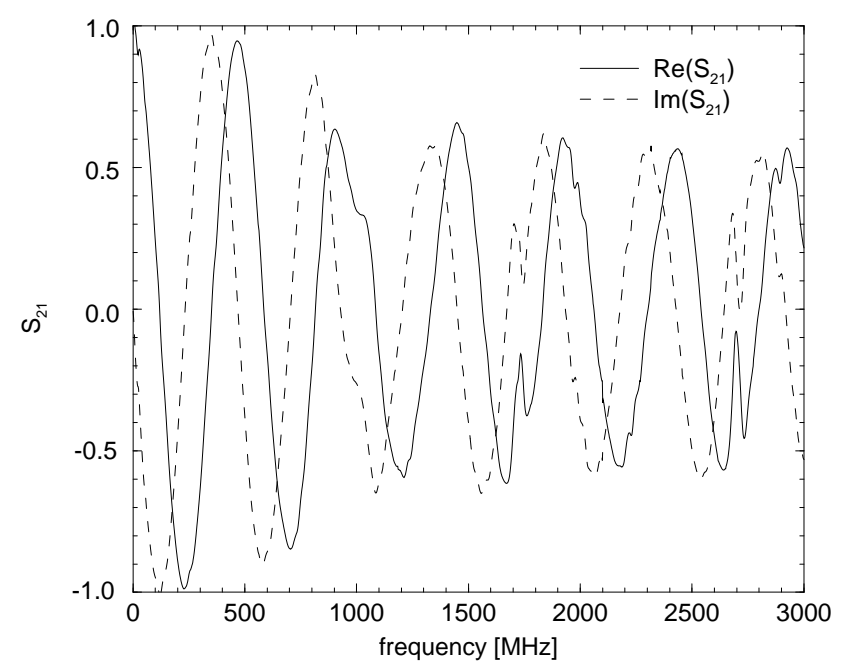

Figure. 4. Calibrated $S_{21}$ Parameter of the Kicker Half Module

The cutoff frequency of the RHIC standard beampipe of diameter $6.91 \mathrm{~cm}$ is $3.3 \mathrm{GHz}$. Therefore the kicker impedance is measured up to $3 \mathrm{GHz}$. Figure 3 gives a plot of the uncalibrated transmission coefficient $S_{21}$ of the kicker and Figure 4 gives the transmission coefficient $S_{21}$ after TRL calibration.

Figure 5 gives a plot of the measured impedance up to a

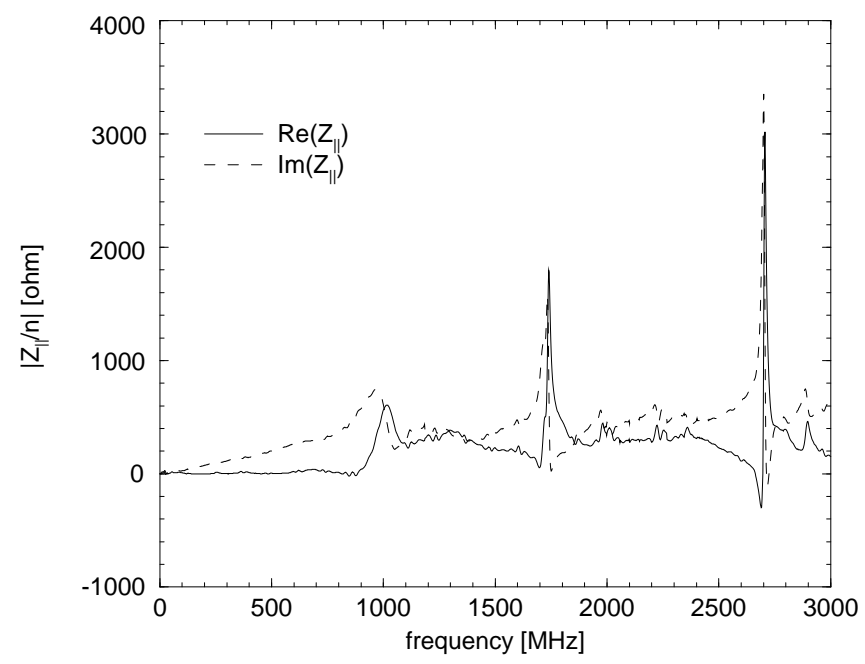

Figure. 5. Injection Kicker Half Module Impedance

frequency of $3 \mathrm{GHz}$. The half length kicker has an inductive impedance with an inductance of 63 nHenry. Therefore the total contribution to the longitudinal broadband impedance $\left|Z_{||} / n\right|$ from all 4 units is .25 ohms. Note also that the impedance shows resonances at high frequencies, between 1 and $3 \mathrm{GHz}$. The frequency $\mathrm{f}$, shunt impedance $R_{s h}$, and $\mathrm{Q}$ of the resonances are given in Table I. Although the ferrite is lossy at high frequencies, the resonances are not completely damped. These resonances are presumed to be higher order TM-like modes of the kicker. The frequency of the resonances is high enough and the $\mathrm{R}$ is low enough so as not to be fatal to the beam. In RHIC, the microwave instability sets a limit on $\left|Z_{\|} / n\right|$ for gold ions at transition crossing to be $1.5 \mathrm{ohm}$. At present, the kickers make a dominant contribution to the machine broadband impedance [6].

Table I

Longitudinal Resonances of the Injection Kicker

\begin{tabular}{|c|c|c|c|}
\hline $\mathrm{f}[\mathrm{MHz}]$ & $R_{s h}[\Omega]$ & $\mathrm{Q}$ & $R_{s h} / Q[\Omega]$ \\
\hline 1000 & 607 & 9 & 67 \\
1740 & 1816 & 83 & 22 \\
2704 & 3004 & 208 & 14 \\
\hline
\end{tabular}

\section{Impedance Reduction}

The following two methods for impedance reduction are being explored:

\section{A. Conductive Coating}

The inside surface of the ceramic beam tube is coated with a palladium/silver conductive paste. As described in [4] and [5], the surface resistivity of the paste is selected so that the total resistance of the coating is $20 \mathrm{ohm}$, and the measured change in kicker risetime is $5 \mathrm{nsec}$. Figure 6 gives a plot of the impedance with the coated ceramic pipe. The resonances are completely damped, and the broadband $\left|Z_{||} / n\right|$ is reduced to $.012 \mathrm{ohm}$. The total broadband impedance from all 4 units is .1 ohm, reduced by a factor of 3 . However, with the high conductive coating, the kicker shows a breakdown at a voltage much lower than its 


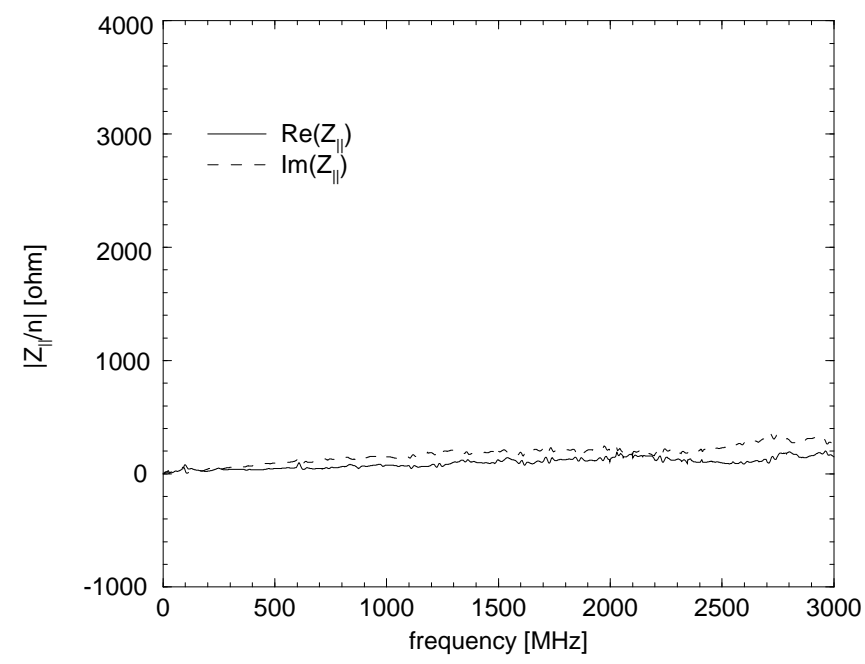

Figure. 6. Kicker Impedance with Conductively Coated Ceramic Pipe

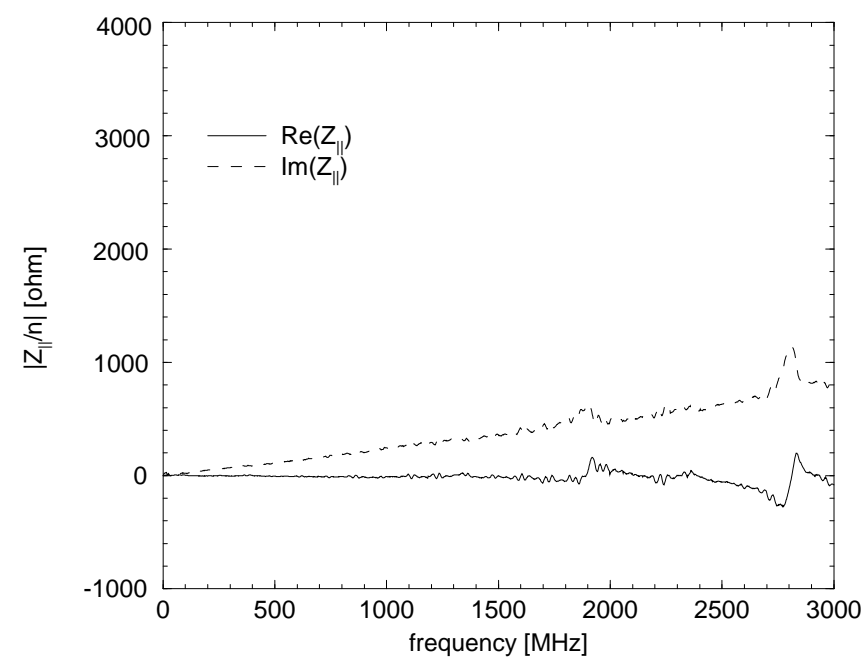

Figure. 7. Kicker Impedance with Copper Strips

nominal operating voltage of $60 \mathrm{kV}$. Therefore, this solution to the impedance reduction problem is disfavored.

\section{B. Copper Strips}

Two copper strips of width $.5 \mathrm{~cm}$ and thickness 3 mil were placed inside the ceramic pipe, at the left and the right sides (Figure 1), along the length of the kicker. Figure 7 shows a plot of the kicker impedance with the strips. The resonances have been damped substantially, and the broadband impedance $\left|Z_{||} / n\right|$ is $.018 \mathrm{ohm}$. The total broadband impedance from all 4 units is $.14 \mathrm{ohm}$, reduced by a factor of 1.8. The copper strips show no significant effect on the field risetimes. With the strips in place, the kicker breaks down at just below its nominal operating voltage. With some slight modifications to the kicker, the strips could be possibly used to reduce impedance.

\section{Conclusion}

The kicker impedance shows resonances between 1 and $3 \mathrm{GHz}$. The frequencies of these resonances are high enough and the $\mathrm{R}$ is low enough so as not to affect the beam. With a broadband impedance of $.26 \mathrm{ohm}$, the injection kickers make a dominant contribution to the broadband impedance. The high conductivity coating damps the resonances completely and reduces the broadband impedance by a factor of 3 , but there is breakdown at relatively low voltage. The copper strips damp the resonances, and reduce the broadband impedance by almost a factor of 2 . However, these also show some breakdown behavior at high voltage. We are currently working on resolving the breakdown problem, and the strips could possibly be used as a solution to the impedance reduction problem.

\section{Status}

Work is in progress with $\mathrm{H}$. Hahn to understand the measured resonances better.

\section{Acknowledgements}

The authors would like to thank M. Harrison, P. Colestock and F. Caspers for helpful discussions. We would also like to thank members of the RHIC Instrumentation Group, RF Group and K. Hartmann, C. Pappas and J. Tuozzolo for their help with measurements.

\section{References}

[1] E. B. Forsyth, "RHIC Injection Kicker", unpublished, December 1992.

[2] "RHIC Design Manual", May 1994.

[3] V. Mane, T. Shea, PAC, May 1993, pp. 3438-3440.

[4] S. Kurennoy, PAC, May 1993, pp. 3420-3422.

[5] A. Piwinski, IEEE Trans Nuclear Science, Vol.NS-24, No. 3, June 1977, pp. 1364-1366.

[6] W. W. MacKay et al., "Estimation of Collective Instabilities In RHIC", these proceedings. 